\title{
In Vitro Culture as a Stressful Factor Triggers Changes in Polyphenols, Flavonoids and Antioxidant Activity in Somatic Hybrids between Solanum tuberosum and S. bulbocastanum and their Respective Parents
}

\author{
Daniel CRUCERIU ${ }^{1}$, Imola MOLNAR ${ }^{1}$, Zorita DIACONEASA ${ }^{2}$, \\ Adriana AURORI ${ }^{1}$, Carmen SOCACIU ${ }^{2}$, Elena RAKOSY-TICAN ${ }^{1 *}$
}

\author{
IBabes-Bolyai University, Faculty of Biology and Geology, Plant Genetic Engineering Group, 5-7 Clinicilor, 400006 Cluj-Napoca, Romania; \\ dani_cruceriu@yahoo.com;molnar.imola5@gmail.com; aaurori2002@gmail.com; elena.rakosy@ubbcluj.ro(*correspondingauthor) \\ ${ }^{2}$ University of Agricultural Sciences and Veterinary Medicine, 3-5 Calea Mănăştur, 400372 Cluj-Napoca, \\ Romania; zorita.sconta@usamvcluj.com; carmen.socaciu@usamvcluj.com
}

\begin{abstract}
Phenols, important secondary metabolites in plants, are responsible for specific defence mechanisms against abiotic stress, due to their strong antioxidant activity. Flavonoids, as part of phenolic group, are also involved in plant stress responses, being primarily responsible for photo-protection against UV solar radiation. Based on these premises, the plant response to optimized in vitro culture was evaluated, by quantifying the total polyphenolic content, the total flavonoid content and the antioxidant activity, both under in vitro and ex vitro conditions. Four closely related potato genotypes were analysed: the wild species Solanum bulbocatanum, S. tuberosum cv. 'Rasant' and two somatic hybrids between them. For all genotypes, both total polyphenolic content and antioxidant activity were increasing under optimized in vitro culture. The shoot responses were genotype dependent and the two somatic hybrids were intermediate between the parents both as morphology and reaction to in vitro stress. The somatic hybrid $1508 / 5$, having morphology similar to potato, was reacting as the wild species, while the somatic hybrid 1508/2, with a similar morphology to the wild species was reacting as potato to in vitro stress. The somatic hybrid 1508/5 being also resistant to late blight is of interest for further use in pre-breeding. Total flavonoid content is decreasing under in vitro as compared to ex vitro conditions, UV-B radiation, the major trigger of flavonoid biosynthesis being absent in the fluorescent light. This study reveals the effect of in vitro culture on flavonoid content and details aspects of the biochemical parameters involved in plant in vitro stress.
\end{abstract}

Keywords: biochemical parameters, electrofusion, optimized in vitro conditions, protoplasts, fusion hybrids, stress factors

Abbreviations: AA - Antioxidant activity; SH - Somatic hybrid; TFC - Total flavonoid content; TPC - Total polyphenolic content

\section{Introduction}

Stress, according to the state-change concept (Strasser, 1988), is represented by any modification of an environmental factor, biotic or abiotic, which forces a biological system away from its optimal state. The presence of a stressful factor generates genetic and epigenetic changes, especially modification at the gene expression level and as a result morphological, anatomical and physiological responses usually occur (Cramer et al., 2011). One of the first such events happening at the cellular level is generation of reactive oxygen species (ROS), which alter gene expression and enzyme activity. ROS also have a role as signalling molecules for initiating defence responses (Mittler et al., 2011). Even though accumulation of ROS is an essential part of the signalling pathways used by the cell to make suitable adjustments in response to environmental changes, they are also harmful for the cell, producing oxidative stress and damaging important cellular components like lipids, proteins or nucleic acids (Foyer and Noctor, 2005). In this context, the quantity of intracellular ROS has to be controlled and that is achieved through the antioxidant system (with its enzymatic and non-enzymatic components), which protects the cell by scavenging ROS (Shao et al., 2008).

One of the major classes of compounds, part of the nonenzymatic antioxidant system, playing an important role in 
76

maintaining the redox state of the cell are the phenolic compounds, due to their strong antioxidant capacity. Phenols may be found constitutively in plant cells, but it was demonstrated that their quantity is rapidly increasing, by de novo synthesis, in the presence of a stressful factor (JacoboVelázquez and Cisneros-Zevallos, 2012; Mierziak et al., 2014). In this way, phenolic compounds are directly involved in the plant defence mechanisms and in overcoming abiotic stress conditions (Ramakrishna and Ravishankar, 2011).

Among polyphenols, flavonoids are well known to have important roles in exceeding a wide range of stressful factors, but these compounds are primarily involved in photoprotection against short-wavelength UV-B solar radiation (over 280-315 nm waveband) (Agati and Tattini, 2010). The UV-B radiation, like all the environmental stressors, enhances the production of ROS at the cellular level, thus damaging cellular components. In response, plants synthesize higher levels of flavonoids (Sisa $e t$ al., 2010), light being a major trigger of flavonoid biosynthesis. Moreover, a positive correlation between the total flavonoid content and the UV radiation was previously demonstrated (Caldwell, 1971). Flavonoids in plants act as sunscreens by absorbing the UV-B radiation and thus reducing the risk of ROS generation, but also as antioxidants by scavenging ROS once they are formed (Agati and Tattini, 2010). All these reports support the assumption that the total flavonoid content in plants is highly related to the UV-B radiation.

In vitro culture implies cultivation of shoots under special conditions regarding humidity, temperature, light exposure, aseptic environment and sucrose/nutrient containing medium. It is well known that, depending on these environmental influences, under in vitro conditions, plantlets are exposed to stress and might present abnormal morphology, anatomy or physiology (Hazarika, 2006). In extreme condition aberrant phenotype such as habituated and hyperhydric ('vitrified') explants might result (Balen et al., 2009). One of the major causes for such phenomena is thought to be the oxidative stress damage occurring in plant tissues during explant preparation and cultivation (Cassells and Curry, 2001). In this context and taking into consideration the state-change concept, one can state that in vitro culture might be considered as a stressful factor for the shoots. However, all these external factors are usually well controlled and optimized for rapid growth and proper development of the explants. To achieve these goals, for each species subject to in vitro culture, specific optimal conditions have been identified, under which plantlets appear healthy and normal (Thorpe, 2007). The questions that remain are if under optimized in vitro culture conditions the explants are still subjected to stress and if so, how their cellular physiology is affected by it.

Pointing out how the in vitro culture influences the plantlets development and physiology is extremely relevant in many experimental designs. First of all, for all the studies being performed on in vitro cultivated shoots, with the main purpose to quantify different primary or secondary metabolites specific for a particular species/genotype, scientists must take into consideration the percentage of such compounds synthesized due to the environmental conditions. Secondly, for the studies that aim to characterize one or more species at the transcriptomic level, major differences between in vitro - ex vitro conditions may occur (Joyce et al., 2003). Last but not least, in the experiments focused on identifying the differences between genetically modified plants and their original counterparts, as part of the risk assessment strategy, an important percentage of the dissimilarities observed are caused by the in vitro procedure (Montero et al., 2011; Fonseca et al., 2015).

Plant responses to environmental stress, including accumulation of phenolic compounds, depend on the duration, severity and number of exposures to the stress factors, but also on plant characteristics, such as the tissue/organ in question, the stage of development or the genotype (Gaspar $e t$ al., 2002). The genotype is a major factor regarding plant stress responses, thus even closely related genotypes might present significant differences in their reactions to stress. Asymmetric somatic hybrids obtained through protoplast electrofusion, are hybrids in which the nuclear genome consists of the full somatic complement of recipient fusion parent and a variable number of chromosomes from the donor one. These hybrids prove to be relevant resources for plant breeders due to their genetic make-up, several desirable genes being transferred from one fusion partner to another. Because of their atypical genetic constitution, their phenotypic traits are unpredictable, several outcomes being possible: the somatic hybrids might be inferior, intermediate or superior to their genitors from a specific phenotypic point of view (Shankar et al., 2013). Therefore, we investigated the total phenolic and flavonoid content as well as the antioxidant activity under in vitro and ex vitro conditions for four different genotypes of Solanum: two asymmetric somatic hybrids $(\mathrm{SH})$ and their parents. The aim of this study was to evaluate the specific response of those related genotypes to in vitro conditions in comparison to plants grown ex vitro at the level of polyphenol and flavonoid biosynthesis as well as antioxidant activity. Those data were correlated to previous results on cytogenetics, morphology and presence of two resistance genes to late blight/ resistant phenotype for better characterization of the somatic hybrids that are recommended for a pre-breeding program.

\section{Materials and Methods}

\section{Plantmaterial}

Four different genotypes of Solanum were tested in this study: a wild diploid Mexican species $S$. bulbocastanum GLKS31741 [blb41, Gross Lüsewitz Potato Collections (GLKS) of the IPK Gene Bank, Leibniz - Institute of Plant Genetics and Crop Plant Research, Germany]; a commercial potato $S$. tuberosum cv. 'Rasant' (Nordring-Kartoffelzucht-und Vermehrungs-GmbH Gross Lüsewitz, Germany); two asymmetric somatic hybrids $-1508 / 5$ and $1508 / 2$, produced by mesophyll protoplast electrofusion. Prior to this study, the hybridity of the plants was demonstrated through flowcytometry and SSR markers, while the asymmetric status of the two somatic hybrids was demonstrated using AFLP markers (Rakosy-Tican et al., 2015). Furthermore, the somatic hybrid $1508 / 5$ proved to be resistant to late blight when tested in detached leaf assay (in two consecutive years) and a field, possessing two late blight resistance genes, Rpi-blb1 and Rpiblb3, known to induce durable resistance to this extremely adaptable and devastating pathogen (Rakosy-Tican, under publication). 
Morphologically, the two somatic hybrids have intermediate phenotype when compared to the parents regarding their height and leaf morphology, as shown in Fig. 1. The $1508 / 5$ somatic hybrid is more similar to the cultivated potato, while the 1508/2 hybrid is similar to $S$. bulbocastanum.

Six individuals for all four genotypes were cultivated in vitro, under controlled optimal environmental conditions for Solanum species [temperature: $21^{\circ} \mathrm{C}$; photoperiod: $16 \mathrm{~h} / 8 \mathrm{~h}$ (light/dark); PPF - photosynthetic photon flux levels (light intensity): $135 \mu \mathrm{mol} \mathrm{m}^{-2} \mathrm{~s}^{-1}$; light spectrum: $380-680 \mathrm{~nm}$ ], on RMB5 medium [Duchefa Netherland - standard MS medium (Murashige and Skoog, 1962) enriched with Gamborg B5 vitamins (Gamborg et al., 1968), 0.7\% agar, $\mathrm{pH}=5.8$ ], for four weeks. The vessels used for the in vitro culture were clear glass jars, equipped with caps with sponges that allow the gas exchange. Each jar contained $100 \mathrm{ml}$ RMB5 medium and 3 explants/jar were cultured. Three individuals for each genotype were transferred in a single jar and cultivated for an additional 4 weeks under the same in vitro conditions as mentioned before. Simultaneously, three individuals for each genotype were cultivated ex vitro, under uncontrolled environmental conditions (room temperature, $24^{\circ} \mathrm{C} \pm 3{ }^{\circ} \mathrm{C}$, photoperiod: approximately $12 \mathrm{~h}$, in direct sunlight during May), in regular soil, for four weeks, including acclimatization. Therefore, the control group consisted in three individuals for each analysed genotype cultivated under ex vitro conditions, while the group of interest was formed from three individuals for each analysed genotype cultivated under in vitro conditions. Among the three individuals belonging to the same genotype, cultivated either under in vitro or ex vitro conditions, the growth was uniform. For each genotype, the ex vitro cultivated plants had a two to three times fold increase in biomass when compared to the in vitro plants.

\section{Sample extraction}

Each sample was obtained from three individuals belonging to one genotype, under one set of conditions, either in vitro or ex vitro. Therefore, eight samples were analysed in this study. For each sample extraction, the second, third and fourth leaf down from apex was collected from all three individuals

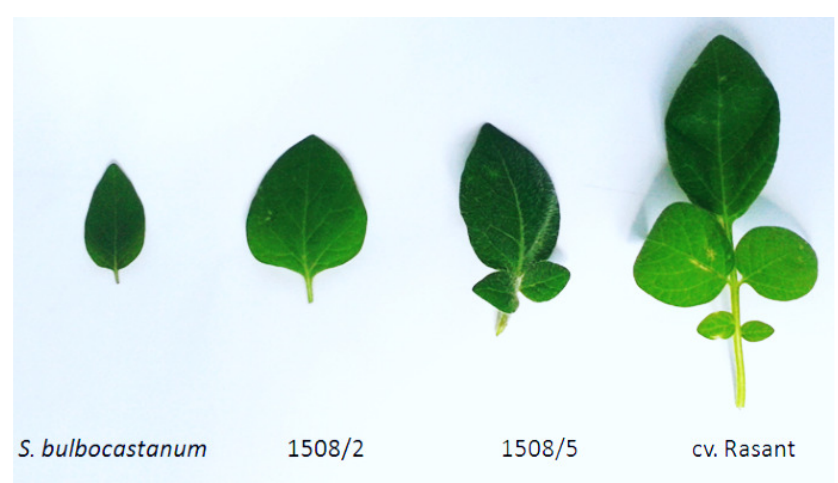

Fig. 1. Comparative leaf morphology of the parents (Solanum bulbocastanum and $S$. tuberosum cv. 'Rasant') and two asymmetric somatic hybrids (1508/2 and 1508/5), after eight weeks ex vitro growth. The third leaf down from apex was used for comparison cultivated under in vitro or ex vitro conditions, belonging to each genotype. All the collected leaves belonging to one sample were milled and $1 \mathrm{~g}$ of the obtained powder was transferred in $10 \mathrm{ml}$ extraction buffer [acidified methanol $-85: 15 \mathrm{v} / \mathrm{v}$, $\mathrm{MeOH}: \mathrm{HCl}$ ) (El-Sayed and Hucl, 2003). The extraction was performed according to the procedure used by Bunea $e t$ al. (2011), through repeated extraction buffer addition, centrifugation and collection, the total extracts for all samples being dried afterwards in a vacuum rotary evaporator (Heidolph Laborota 4000 Efficient), at $40{ }^{\circ} \mathrm{C}$ and re-dissolved in $2 \mathrm{ml}$ extraction buffer, prior to further analysis.

\section{Total polyphenolic and flavonoid content determination}

Total phenolic content (TPC), was determined using the colorimetric Folin-Cicalteu method (Singleton et al., 1999). 25 $\mu \mathrm{L}$ of sample was used for the oxidation step with FolinCicalteu Reagent and a subsequent neutralization step with $\mathrm{Na}_{2} \mathrm{CO}_{3}$. Sample absorbance was measured at $750 \mathrm{~nm}$ through spectrophotometry (BioTek Instruments, Winooski, VT) and the results were expressed as $\mathrm{mg}$ of gallic acid $100 \mathrm{~g}^{-1}$ fresh weight (FW). Each sample was measured three times.

The total flavonoid content (TFC) was evaluated for each sample based on the colorimetric method elaborated by Kim $e t$ al. (2003), by consecutive addition of $5 \% \mathrm{NaNO}_{2}, 10 \% \mathrm{AlCl}_{3}$ and $1 \mathrm{~N} \mathrm{NaOH}$ to each alcoholic extract. The absorbance was measured at $510 \mathrm{~nm}$ through spectrophotometry (JASCO V630 series, International Co.). Each sample was measured three times and the results were expressed as $\mathrm{mg}$ quercetin equivalents $100 \mathrm{~g}^{-1} \mathrm{FW}$. Even though this method does not detect all flavonoid subclasses, it is one of the most widely used procedures to assess the flavonoid content in food and medicinal plant samples. It detects the flavonols and flavones in an extract, two major classes of flavonoids found in plants, but also some phenolic acids, such as the caffeic acid (Pekal and Pyrzynska, 2014). This protocol was chosen in these experiments also because it proved to be more reliable in determining the flavonoid content then other procedures (Pekal and Pyrzynska, 2014).

\section{Antioxidant activity determination}

The antioxidant capacity (AA) was determined through the ABTS assay (ABTS radical cation distaining assay), based on the capacity of the samples to scavenge ABTS radicals, in comparison with Trolox, a standard antioxidant (Arnao et al., 2001). $20 \mu \mathrm{L}$ of each sample extract and Trolox were added in $170 \mu \mathrm{L} \mathrm{ABTS}^{+}$solution, which was prepared $16 \mathrm{~h}$ before the experiment. The absorbance was measured at $734 \mathrm{~nm}$ on a 96 well microplate, through spectrophotometric means (BioTek Instruments, Winooski, VT). The results were expressed as $\mu$ mole Trolox equivalents $100 \mathrm{~g}^{-1} \mathrm{FW}$ and for each genotype 3 measurements were performed.

\section{Statistical analysis}

Unpaired tow-tailed t-tests were used in order to determine if the differences observed in TPC, TFC and AA under in vitro and ex vitro conditions for each genotype are statistically significant. Furthermore, the same statistical analysis was performed to assess the differences between genotypes under $e x$ vitro conditions (control), for each physiological trait. Significance of difference was defined at the $5 \% \operatorname{level}(\mathrm{p}<0.05)$. Previously, our data were tested for their normal distribution 
78

using the D'Agostino-Pearson omnibus normality test and for their variances using the $F$ test. All statistical analyses were performed using the GraphPad Prism software (Graph Pad Software Inc; San Diego, CA, USA). The increased or decreased rate of TPC, TFC and AA (parameters) specific for each genotype between the in vitro and ex vitro (control) conditions was calculated as follows:

Increased / decreased rate $(\%)=[$ (parameter in in vitro / parameter in ex vitro) - 1] x 100.

\section{Results}

\section{Totalpolyphenolic content}

The highest polyphenolic content, both in vitro and ex vitro, was found in the wild species $S$. bulbocastanum, while low TPC was revealed in the commercial $S$. tuberosum cv. 'Rasant' and in the somatic hybrid $1508 / 2$ (Fig. 2). The 1508/5 genotype presents intermediate phenotype between the two parental species in ex vitro conditions regarding the TPC pattern, but because of its low increasing rate, in in vitro culture this hybrid is similar to its cultivated genitor, potato.

By comparing the total polyphenolic content under ex vitro conditions with TPC in in vitro culture, the accumulation of phenolic compounds becomes conspicuous for all four genotypes, even though the increasing rates are various. For all four genotypes, significant differences were found between the two sets of conditions. The highest increasing rates were found in S. tuberosum cv. 'Rasant' and in the 1508/2 hybrid,

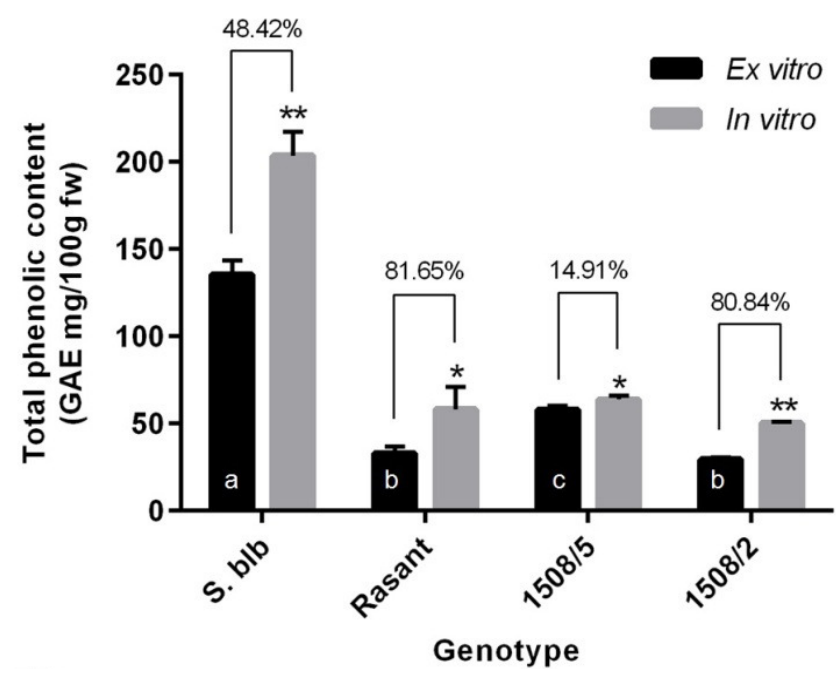

Fig. 2. Total phenolic content under ex vitro (black) and in vitro (gray) conditions in: $S$. bulbocastanum (S. blb), $S$. tuberosum cv. 'Rasant' and two somatic hybrids between them $(1508 / 5$ and $1508 / 2)$. Data are means \pm SD $(n=3)$. For each measurement under in vitro conditions, ${ }^{*}$ shows a statistically significant difference $(\mathrm{p}<0.05)$ and ${ }^{* *}$ shows a highly significant difference $(\mathrm{p}<0.01)$ in TPC, when compared to $e x$ vitro conditions. The increased rates (\%) in TPC under ex vitro conditions for each genotype are shown above each set of data. Significant differences $(\mathrm{p}<0.05)$ between genotypes under $e x$ vitro (control) conditions are represented by different letters genotypes that express the smallest quantities of constitutive polyphenols under ex vitro conditions. On the other hand, the somatic hybrid 1508/5, which synthesizes almost double quantities of constitutive phenolic compounds as compare to $S$. tuberosum cv. 'Rasant', it's characterized by the lowest growth rate, which translates into the smallest accumulation of TPC.

\section{Total flavonoid content}

The highest flavonoid content was determined in $S$. bulbocastanum, both ex vitro and in vitro, while the lowest amounts of flavonoids under ex vitro conditions were found in S. tuberosum cv. 'Rasant' (Fig. 3). The $1508 / 5$ somatic hybrid presents intermediate flavonoid quantities when compared with the parents in vitro, as well as under ex vitro conditions.

When comparing the TFC for each genotype under $e x$ vitro (control) and in vitro conditions, statistically significant differences were observed for $S$. bulbocastanum and for both somatic hybrids. However, in contrast with the TPC accumulation and the AA increase in vitro, the total flavonoid content is much higher under ex vitro conditions, not in the in vitro culture, as it might be expected.

\section{Antioxidant activity}

The highest AA, both under ex vitro and in vitro conditions, was revealed in $S$. bulbocastanum. Opposed to the wild species, in ex vitro culture the lowest antioxidant capacity was found in the somatic hybrid 1508/2. On the other hand, in in vitro culture, the cultivated species $S$. tuberosum cv. 'Rasant'

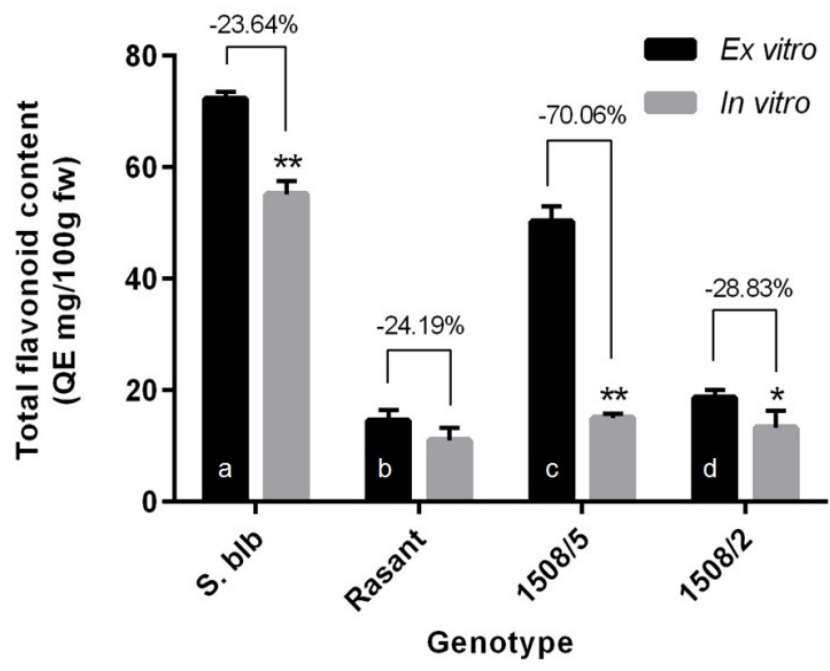

Fig. 3. Total flavonoid content under ex vitro (black) and in vitro (gray) conditions in: S. bulbocastanum (S. blb), $S$. tuberosum cv. 'Rasant' and two somatic hybrids between them $(1508 / 5$ and $1508 / 2)$. Data are means \pm SD $(n=3)$. For each measurement under in vitro conditions, ${ }^{*}$ shows a statistically significant difference $(\mathrm{p}<0.05)$ and ${ }^{* *}$ shows a highly significant difference $(\mathrm{p}<0.01)$ in TFC, when compared to $e x$ vitro conditions. The increased rates (\%) in TFC under ex vitro conditions for each genotype are shown above each set of data. Significant differences $(\mathrm{p}<0.05)$ between genotypes under $e x$ vitro (control) conditions are represented by different letters 
becomes the genotype with the poorest antioxidant activity. Compared to its genitors, the somatic hybrid $1508 / 5$ holds an intermediate antioxidant capacity, both in ex vitro and in in vitro culture.

Except $S$. bulbocastanum, all the other genotypes present an increased antioxidant potential under in vitro conditions, with statistically significant differences $(\mathrm{p}<0.01)$ when compared with the AA under ex vitro conditions. Between these 3 genotypes, the lowest increasing rate occurs in $S$. tuberosum cv. 'Rasant', while the highest is characteristic for the 1508/2 hybrid, which is doubling its antioxidant capacity under in vitro conditions.

\section{Discussion}

Based on our results, it can be noted that both under in vitro or ex vitro conditions, the highest polyphenol content, flavonoid content and the greatest antioxidant activity occur in S. bulbocastanum. This wild species has effective resistance mechanisms to different abiotic stress factors, but also to some biotic agents, like aphids (Alvarez et al., 2006), nematodes (Austin et al., 1993) or fungi (Lokossou et al., 2010). In this context, higher TPC, TFC and AA were actually expected for the wild species, all three physiological traits taken into consideration being actively involved in the resistance mechanisms (Lattanzio et al., 2006; Mierziak et al., 2014).

The SH 1508/5 had intermediate TPC, TFC or AA when compared to its parents, while $\mathrm{SH} 1508 / 2$ was similar in biochemical patterns with $S$. tuberosum cv. 'Rasant'. The two

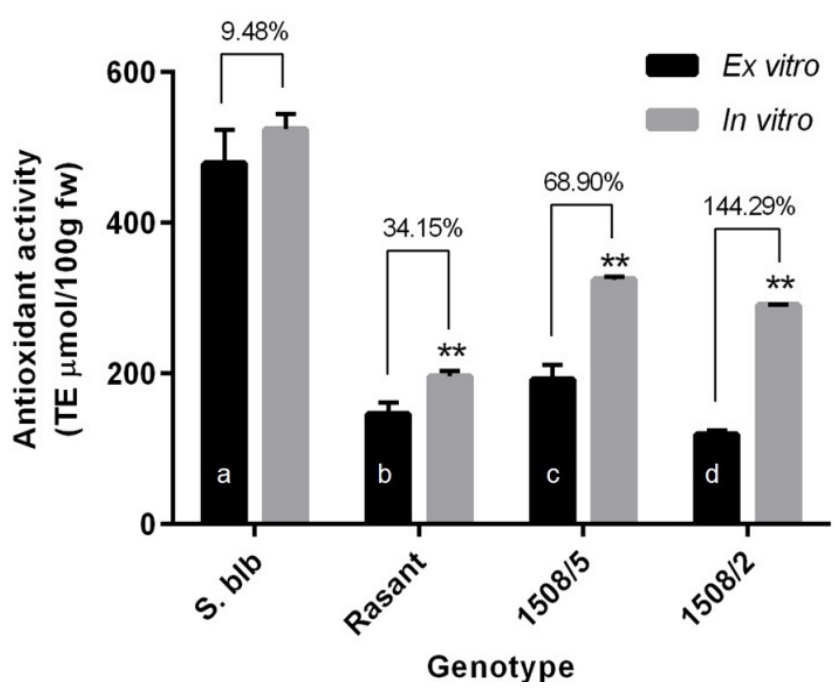

Fig. 4. Antioxidant activity under ex vitro (black) and in vitro (gray) conditions: $S$. bulbocastanum (S. blb), S. tuberosum cv. 'Rasant' and two somatic hybrids between them (1508/5 and $1508 / 2)$. Data are means \pm SD $(n=3)$. For each measurement under in vitro conditions, ${ }^{*}$ shows a statistically significant difference $(\mathrm{p}<0.05)$ and ${ }^{* *}$ shows a highly significant difference $(\mathrm{p}<0.01)$ in AA, when compared to ex vitro conditions. The increased rates (\%) in AA under ex vitro conditions for each genotype are shown above each set of data. Significant differences $(\mathrm{p}<0.05)$ between genotypes under ex vitro (control) conditions are represented by different letters somatic hybrids are asymmetric hybrids and thus their genetic makeup consists of two different parental genomes which have suffered uni- or bidirectional chromosome losses (RakosyTican et al., 2015). Therefore their phenotype with regard to TPC, TFC and AA was unpredictable. A relevant aspect that has to be highlighted is that the $\mathrm{SH} 1508 / 5$ acquired new traits (higher TPC, TFC and AA) when compared to the cultivated potato. Corroborating these findings with previous data according to which this hybrid has gained resistance to Phythophtora infestans due to the presence of Rpi-blb1 and Rpiblb3 genes in its genome (Rakosy-Tican, under publication), we can state that this hybrid has great potential for inclusion in future breeding programs.

The phenotypic appearance of the somatic hybrids were both intermediate between the two parental species, but $\mathrm{SH}$ $1508 / 2$ was much similar to $S$. bulbocastanum, while $\mathrm{SH}$ $1508 / 5$ had leaf morphology similar to the cultivated potato $\mathrm{Cv}$. 'Rasant'. The morphological features did not correspond in their similarity to biochemical characteristics, as shown before. However, the fact that the $1508 / 5$ hybrid is more similar with $S$. bulbocastanum in regard to the resistance mechanisms and more alike $S$. tuberosum from a morphological perspective, strengthens this hybrid's potential for future potato breeding.

According to our results, optimized in vitro conditions represent a stressful factor for the plantlets. Plants respond to environmental stress by activating a number of defence mechanisms, including accumulation of phenolic compounds and increased antioxidant activity (Jacobo-Velázquez and Cisneros-Zevallos, 2012; Ramakrishna and Ravishankar, 2011). For all four analysed genotypes, both the polyphenol quantities and the antioxidant activity increase under in vitro conditions. These results demonstrate that in vitro conditions activate the plants defence mechanisms, by de novo synthesis of polyphenols, due to which an increased antioxidant capacity is observed. These results complement previous studies, which were based on gene expression using microarray analysis and metabolomics, showing that the in vitro tissue culture is perceived as a stressful factor by the explants (Desjardins $e t$ al., 2009).

Several studies reported in the literature are focused on creating a stressful environment under in vitro explant culture, in order to improve the synthesis of important secondary metabolites (Patel and Krishnamurthy, 2013) or antioxidants (Matkowski, 2008), to assay the effects of abiotic stresses on different species (Terletskaya and Khailenko, 2014), to improve the efficiency of organogenesis in plant in vitro culture (Adamczuk et al., 2012) or to develop biotechnological tools for crop breeding and in vitro stress selection (Pérez-Clemente and Gómez-Cadenas, 2012). None of these studies is taking into account the stress factor represented by the optimized in vitro culture itself.

The total flavonoid content was the only physiological trait examined in this study that decreased under in vitro conditions. Light is one of the major environmental factors that influences the flavonoid de novo biosynthesis, primarily short-wavelength UV solar radiation (Tegelberg et al., 2001; Ryan et al., 2002). The in vitro explants were grown in the absence of UV-B radiation because the light used had wavelengths in the range of 380-680 nm (Philips lamps - see: http://www.lighting. philips.com). On the other hand, the ex vitro plants were cultivated in direct sunlight, during May, where UV-B 
80

radiation was present. Previous studies (Sisa et al., 2010; Agati and Tattini, 2010), proving that flavonoid biosynthesis is strongly related with the presence and amount of UV-B radiation, support our results. The UV-B radiation present in the direct sunlight is activating the flavonoid biosynthesis, while the optimized in vitro culture produces the accumulation of polyphenols, others then flavonoids and increases the antioxidant activity. These results highlight the active involvement of other polyphenols then flavonoids in plant responses to in vitro stress.

Plant responses to environmental stress depend, among others, on the genotype of the organism (Gaspar et al., 2002). Thus, the accumulation of phenolic compounds or flavonoids is also thought to be genotype dependent (Al-Saikhan et al., 1995). The present study shows that, even in the case of four closely related organisms ( 2 species belonging to the same genus and their somatic hybrids), differences in phenolic compounds accumulation are present under in vitro conditions, both quantities and increasing rates being specific for each genotype. It was noticed that genotypes with the lowest levels of constitutive phenolic compounds under ex vitro conditions, namely $S$. tuberosum cv. 'Rasant' and the SH 1508/2, had much higher increasing rates. These results are consistent with previous reported data. Studies carried out on lettuce demonstrate that tissue with small quantities of polyphenols had a higher phenolic accumulation in response to heat shock stress (Saltveit, 2000). The results presented here on potato somatic hybrids with the sexually incompatible species Solanum bulbocastanum might be applied to other wild species for better understanding of in vitro stress on plants with unique genetic constitutions and further application in breeding.

\section{Conclusions}

In this study the constitutive phenolic content, flavonoid content and the antioxidant activity of the potato cultivar $S$. tuberosum cv. 'Rasant', the wild species $S$. bulbocastanum, and two of their somatic hybrids, $1508 / 5$ and $1508 / 2$ were quantified in order to ask two questions:

1) Are there differences between related genotypes in their response to in vitro stress?

2) Which is the somatic hybrid that is most suited to be further used in a pre-breeding program?

The answers that can be drowning from the results are:

1) There are differences between the four genotypes analysed, in relation to polyphenol accumulation and antioxidant activity triggered by in vitro culture, but surprisingly the flavonoids analysed are reduced in vitro due to low level UV-B present in the fluorescent light;

2) The somatic hybrid $1508 / 5$ proved to be superior when compared with the cultivated potato from the points of view of reaction to in vitro stress, antioxidant activity and previous results on late blight resistance and thus is under consideration for inclusion in a pre-breeding program.

\section{Acknowledgements}

The work of E R-T and I E-M was supported by the Romanian Authority of Research project CNCS UEFISCDI
PNII-ID-PCE-2011-3-0586. The authors acknowledge Dr. Ramona Thieme for providing preserved parents and somatic hybrids, produced with E R-T, and used in this study.

\section{References}

Adamczuk A, Siegień I, Ciereszko I (2012). Morphogenesis of plants in vitro under stress conditions. In: Łaska G(Eds). Biological diversity - from cell to ecosystem. Polish Botanical Society-Branch in Bialystokpp 25-40.

Agati G, Tattini M (2010). Multiple functional roles of flavonoids in photoprotection. New Phytologist 186:786-793.

Al-Saikhan MS, Howard IR, Miller JC Jr (1995). Antioxidant activity and total phenolics in different genotypes of potato (Solanum tuberosum, L.). Journal of Food Science 60(2):341-343.

Alvarez AE, Tjallingii WF, Garzo E, Vleeshouwers V, Dicke M, Vosman B (2006). Location of resistance factors in the leaves of potato and wild tuber bearing Solanum species to the aphid Myzuspersicae. Entomologia Experimentalis et Applicata 121(2):145-157.

Arnao MB, Cano A, Alcolea JF, Acosta M (2001). Estimation of free radicalquenching activity of leaf pigment extracts. Phytochemical Analysis 12:138-143.

Austin S, Pohlman JD, Brown CR, Mojtahedi H, Santo GS, Douches DS, Helgeson JP (1993). Interspecific somatic hybridization between Solanum tuberosum and S. bulbocastanum dun. as means of transferring nematode resistance. American Journal of Potato Research 70:485-495.

Balen B, Tkalec M, Pavokovic D, Pevalek-Kozlina B, Krsnik-Rasol M (2009). Growth conditions in in vitro culture can induce oxidative stress in Mammillaria gracilis tissues. Journal of Plant Growth Regulation 28:36-45.

Bunea A, Dugină DO, Pintea AM, Sconța Z, Bunea CI, Socaciu C (2011). Comparative polyphenolic content and antioxidant activities of some wild and cultivated blueberries from Romania. Notulae Botanicae Horti Agrobotanici39(2):70-76.

Caldwell MM (1971). Solar UV irradiation and the growth and development of higher plants. Photophysiology 6:131-177.

Cassells AC, Curry RF (2001). Oxidative stress and physiological, epigenetic and genetic variability in plant tissue culture: implications for micropropagators and genetic engineers. Plant Cell, Tissue and Organ Culture 64:145-157.

Cramer GR, Urano K, Delrot S, Pezzotti M, Shinozaki K (2011). Effects of abiotic stress on plants: a systems biology perspective. BMC Plant Biology 11:163-177.

Desjardins Y, Dubuc J-F, Badr A (2009). In vitro culture of plants: a stressful activity! Acta Horticulturae 812:29-50.

El-Sayed MAA, Hud P (2003). Composition and stability of anthocyanins in blue-grained wheat. Journal of Agriculture and Food Chemistry 51:2174-2180.

Fonseca C, Planchon S, Serra T, Chander S, Saibo NJM, Renaut J, Oliveira $\mathrm{MM}$, Batista $\mathrm{R}$ (2015). In vitro culture may be the major contributing factor for transgenic versus nontransgenic proteomic plant differences. Proteomics 15:124-134.

Foyer $\mathrm{CH}$, Noctor $\mathrm{G}$ (2005). Redox homeostis and antioxidant signaling: A metabolic interface between stress perception and physiological responses. Plant Cell 17:1866-1875. 
Gamborg OL, Miller RA, Ojima K (1968). Nutrient requirements of suspension culture of soybean root cells. Experimental Cell Research 50:151-158.

Gaspar T, Franck T, Bisbis B, Kever C, Jouve L, Hausman JF, Dommes J (2002). Concepts in plant stress physiology. Application to plant tissue cultures. Plant Growth Regulation 37:263-285.

Jacobo-Velázquez DA, Cisneros-Zevallos L (2012). An alternative use of horticultural crops: Stressed plants as biofactories of bioactive phenolic compounds. Agriculture-London 2:259-271.

JoyceSM, Cassells AC, Jain SM (2003). Stress and aberrant phenotypes in in vitro culture. Plant Cell, Tissue and Organ Culture 74:103-121.

Hazarika BN (2006). Morpho-physiological disorders in in vitro culture of plants. ScientiaHorticulturae 108:105-120.

Kim DO, Jeong SW, Lee CY (2003). Antioxidant capacity of phenolic phytochemicals from various cultivars of plums. Food Chemistry 81:321-326.

Lattanzio V, Lattanzio VMT, Cardinali A (2006). Role of phenolics in the resistance mechanisms of plants against fungal pathogens and insects. Phytochemistry: Advances in Research 37:23-67.

Lokossou AA, Rietman R, Wang M, Krenek P, van der Schoot H, Henken B, ... Vosman B (2010). Diversity, distribution, and evolution of Solanum bulbocastanum late blight resistance genes. Molecular PlantMicrobe Interactions 23:1206-1216.

Matkowski A (2008). Plant in vitro culture for the production of antioxidants - A review. Biotechnology Advances 26:548-560.

Mierziak J, Kostyn K, Kulma A (2014). Flavonoids as important molecules of plant interactions with the environment. Molecules 19:1624016265.

Mittler R, Vanderauwera S, Suzuki N, Miller G, Tognetti VB, Vandepoele K, Gollery M,Shulaev V, Van BF (2011). ROS signaling: the new wave? Trends in Plant Science 16(6):300-309.

Montero M, Coll A, Nadal A, Messeguer J, Pla M (2011). Only half the transcriptomic differences between resistant genetically modified and conventional rice are associated with the transgene. Plant Biotechnology Journal 9:693-702.

Murashige T, Skoog F (1962). A revised medium for rapid growth and bio assays with tobacco tissue cultures. Physiologia Plantarum 15(3):473497.

Patel H, Krishnamurthy R(2013). Elicitors in plant tissue culture. Journal of Pharmacognosy and Phytochemistry 2:60-65.

Pérez-Clemente R, Gómez-Cadenas A (2012). In vitro tissue culture, a tool for the study and breeding of plants subjected to abiotic stress conditions. In: Leva A, Rinaldi LMR (Eds). Recent advances in plant in vitro culture. InTech pp 91-108.

Philips (2016). TL-D Standard colours. Retrived 2016 February 1 from http://www.lighting philips.com/main/prof/lamps/fluorescent-lampsand-starters/tl-d/tl-d-standardcolours/928025405323_EU/product.
Pekal A, Pyrzynska K (2014). Evaluation of aluminium complexation reaction for flavonoid content assay. Food Analytical Methods 7:17761782.

Rakosy-Tican E, Thieme R, Nachtigall M, Molnar I, Denes TE(2015). The recipient potato cultivar influences the genetic makeup of the somatic hybrids between five potato cultivars and one cloned accession of sexually incompatible species Solanum bulbocastanum Dun. Plant Cell, Tissue and Organ Culture 122(2):395-407.

Ramakrishna A, Ravishankar GA (2011). Influence of abiotic stress signals on secondary metabolites in plants. Plant Signaling and Behavior 6(11):1720-1731.

Ryan KG, Swinny EE, Markham KR, Winefield C (2002). Flavonoid gene expression and $\mathrm{UV}$ photoprotection in transgenic and mutant Petunia leaves. Phytochemistry 59:23-32.

Reyes LF, Cisneros-Zevallos L (2003). Wounding stress increases the phenolic content and antioxidant capacity of purple-flesh potatoes (Solanum tuberosum L.). Journal of Agriculture and Food Chemistry 51:5296-5300.

Saltveit ME (2000). Wound induced changes in phenolic metabolism and tissue browning are altered by heat shock. Postharvest Biology and Technology 21:61-69.

Shankar LP, Eeckhaut T, Dieter D, Van Bockstaele E, Van HuylenbroeckJ (2013). Asymmetric Somatic Plant Hybridization: Status and Applications. American Journal of Plant Sciences 4:1-10.

Shao B, Chu L-Y, Lu Z-H, Kang C-M (2008). Primary antioxidant free radical scavenging and redox signaling pathways in higher plant cells. International Journal of Biological Sciences 4(1):8-14.

Singleton VL, Orthofer R, Lamuela-Raventós RM, Lester P (1999). Analysis of total phenols and other oxidation substrates and antioxidants by means of Folin-Ciocalteu reagent. Methods in Enzymology 299:152178.

Sisa M, Bonnet SL, Ferreira D, Van der Westhuizen JH (2010). Photochemistry of flavonoids. Molecules 15:5196-5245.

Strasser RJ (1988). A concept for stress and its application in remote sensing. In: Lichtenthaler HK (Eds). Applications of chlorophyll fluorescence. Kluwer Academic Publishers, Dordrecht pp 333-337.

Tegelberg R, Julkunen-Tiitto R, Japhal P (2001). The effects of long-term elevated UV-B on the growth and phenolics of field-grown silver birch (Betulapendula). Global Change Biology 7:839-848.

Terletskaya N, Khailenko N (2014). Tissue culture in vitro as a model system for studying the effects of abiotic stresses on different species of wheat. In: Mastorakis $\mathrm{N}$ el al. (Eds). Advances in environmental technology and biotechnology. WSEAS Press pp 102-107.

Thorpe TA (2007). History of plant tissue culture. Molecular Biotechnology 37:169-180. 